\title{
Hadron-quark phase transition in asymmetric matter with dynamical quark masses
}

\author{
G.Y. Shao, ${ }^{1}$ M. Di Toro, ${ }^{1,2, *}$ B. Liu,${ }^{3,4}$ M. Colonna, ${ }^{1}$ V. Greco, ${ }^{1,5}$ Y.X. Liu,${ }^{6,7}$ and S. Plumari ${ }^{1,5}$ \\ ${ }^{1}$ INFN-Laboratori Nazionali del Sud, Via S. Sofia 62, I-95123 Catania, Italy \\ ${ }^{2}$ Physics and Astronomy Dept., University of Catania, Italy \\ ${ }^{3}$ IHEP, Chinese Academy of Sciences, Beijing, China \\ ${ }^{4}$ Theoretical Physics Center for Scientific Facilities, \\ Chinese Academy of Sciences, Beijing, China \\ ${ }^{5}$ Physics and Astronomy Dept., University of Catania \\ ${ }^{6}$ Department of Physics and State Key Laboratory of \\ Nuclear Physics and Technology, Peking University, Beijing 100871, China \\ ${ }^{7}$ Center of Theoretical Nuclear Physics, \\ National Laboratory of Heavy Ion Accelerator, Lanzhou 730000, China
}

\begin{abstract}
The two-Equation of State (EoS) model is used to describe the hadron-quark phase transition in asymmetric matter formed at high density in heavy-ion collisions. For the quark phase, the threeflavor Nambu-Jona-Lasinio (NJL) effective theory is used to investigate the influence of dynamical quark mass effects on the phase transition. At variance to the MIT-Bag results, with fixed current quark masses, the main important effect of the chiral dynamics is the appearance of an End-Point for the coexistence zone. We show that a first order hadron-quark phase transition may take place in the region $\mathrm{T} \subset(50-80) \mathrm{MeV}$ and $\rho_{B} \subset(2-4) \rho_{0}$, which is possible to be probed in the new planned facilities, such as FAIR at GSI-Darmstadt and NICA at JINR-Dubna. From isospin properties of the mixed phase some possible signals are suggested. The importance of chiral symmetry and dynamical quark mass on the hadron-quark phase transition is stressed. The difficulty of an exact location of Critical-End-Point comes from its appearance in a region of competition between chiral symmetry breaking and confinement, where our knowledge of effective QCD theories is still rather uncertain.

PACS numbers: $12.38 . \mathrm{Mh}, 25.75 . \mathrm{Nq}$
\end{abstract}

\section{INTRODUCTION}

The determination of the phase diagram of strongly interacting matter and the search for signals of the hadronquark phase transition are challenges both in theory and experiment. Intensive studies on these fields have been developed in the last decades [1-15]. Most phase diagrams have been derived from Monte Carlo calculations of Lattice QCD [1, 4, 8] or effective chiral models [5$7,15]$ with quark degree of freedom. In the chiral effective model, one can describe well the line of chiral phase transition and the complicated phase diagram of color superconductivity [16-18], while the confinement phase transition for the gluon part can be investigated with the extended NJL model coupled to the Polyakov loop $[9,12-14,19-22])$ in the temperature and chemical potential plane. However a large uncertainty remains about the derivation of baryon density and energy density of the onset of the hadron-quark phase transition [14, 23].

Although there are attempts to describe nuclear and quark matter in unified effective models [24-28], further investigations are needed to give more satisfying results, in particular at high baryon and isospin densities, of interest for the expected phase transition in heavy ion collisions and compact stars.

An alternative approach to describe the hadron-quark

*Corresponding author: ditoro@lns.infn.it phase transition is based on a two-EoS model with the Gibbs criteria, which has been widely used to make predictions on the phase transition in the interior of neutron stars (e.g., [29-35] ).

We remark that, even in the two-EoS approach, only a few papers have studied the phase diagram of hadronquark transitions at high baryon density in connection to the phenomenology of heavy-ion collision ten $\mathrm{A} \mathrm{GeV}$ range (intermediate energies) [36-40]. In Ref. [36] the phase transition from hadron to quark matter has been firstly analyzed for isospin asymmetric matter. It should be noticed that recently increasing attention is paid to this aspect, and some observable effects are suggested to be seen in charged meson yield ratio and in the onset of quark number scaling of the meson/baryon elliptic flows $[37,38]$. This provides us a new orientation to investigate the hadron-quark phase transition, and can stimulate some new relevant research in the field. Later, hyperons have been included in [39], but the calculated results show that strange baryons are not important due to the exiguous final population in the short time scale of a nucleus-nucleus collision. Furthermore, the Cooper pair effect of $u, d$ quarks (two flavor superconductor, 2SC) has been considered in the quark phase [40], which reduces the symmetry energy difference between hadronic and quark phases. The most important conclusion emerging from these works is that the onset density of hadronquark phase transition is smaller in asymmetric matter than that in symmetric matter, which is possibly reached through heavy-ion reactions at new planned facilities, such as FAIR at GSI-Darmstadt and NICA at JINR- 
Dubna, where heavy ion beams (even unstable, with large isospin asymmetry) will be available with good intensities in the 1-30 A GeV energy region.

One drawback in all these calculations is that current mass (or massless) $u, d$ quarks are taken for the quark phase, where the MIT-Bag like models are used. The obtained results are possibly reliable at high density, after the restoration of chiral symmetry due to the asymptotic freedom of QCD, but the chiral symmetry breaking at finite temperature and low densities is not accounted for. From $\rho \sim 4 \rho_{0}$ to smaller densities, the dynamical masses of $u, d$ quarks become larger and larger, and they almost reach $200 \mathrm{MeV}$ at $\rho \sim \rho_{0}$ for some parameter sets. This means that non-perturbative effects become more and more important with the decreasing baryon density. Therefore, calculations of properties of the phase diagram at low density and finite temperature are not fully consistent. As a matter of fact when the MIT-Bag model is taken for the quark part, we see that the T- $\rho$, T- $\mu$ phase diagrams highly depend on the values of the bag constant $B$, which cannot be determined accurately [38].

In order to obtain more reliable theoretical results and predict possible observables in the planned experiments, we take the Nambu-Jona Lasinio (NJL) model to describe the quark phase with the interaction between quarks, where the chiral symmetry breaking and restoration are well described.

It was also proposed in Ref. [41], that in high energy heavy-ion collisions strange and antistrange quarks can be produced by thermal excitation (with net strangeness being zero required by the conservation law of strangeness in strong interaction) and strangeness would be much more abundant in the quark component. Therefore we will also consider the thermal excitation of strange and antistrange quark, but we need to keep the chemical potential of strange quark to be zero, $\mu_{s}=0$, before the beginning of hadronization in the expanding process to make sure of the net strangeness being zero. One mechanism of hadron production is quark recombination [42-47], which is out of the range of the discussion in this paper.

The paper is organized as follows. In Section II, we describe briefly the used effective Lagrangians and give the relevant formulas for the Relativistic Mean Field ( $R M F)$ theory adopted for the hadron sector. In Section III, we present the calculated phase diagrams and compare them with those obtained in the MIT-Bag model. Besides, we present some discussions and conclusions. Finally, a summary is given in Section IV.

\section{NUCLEAR MATTER, QUARK MATTER AND THE MIXED PHASE}

For the hadron phase, the non-linear Relativistic Mean Field (RMF) approach is used, which provides an excellent description of nuclear matter and finite nuclei. One can calibrate the hadronic equation of state at zero temperature and normal nuclear densities, and then extrapolate into the regime of finite density and temperature. Our parametrizations are also tuned to reproduce collective flows and particle production at higher energies, where some hot and dense matter is probed, see [48] and refs. therein. The exchanged mesons include the isoscalar-scalar meson $(\sigma)$, isoscalar-vector meson $(\omega)$, isovector-vector meson $(\rho)$ and isovector-scalar meson $(\delta)$. The effective Lagrangian can be written as

$$
\begin{aligned}
\mathcal{L}=\bar{\psi} & {\left[i \gamma_{\mu} \partial^{\mu}-M+g_{\sigma} \sigma+g_{\delta} \boldsymbol{\tau} \cdot \boldsymbol{\delta}-g_{\omega} \gamma_{\mu} \omega^{\mu}-g_{\rho} \gamma_{\mu} \boldsymbol{\tau} \cdot \boldsymbol{\rho}^{\mu}\right] \psi } \\
& +\frac{1}{2}\left(\partial_{\mu} \sigma \partial^{\mu} \sigma-m_{\sigma}^{2} \sigma^{2}\right)-\frac{1}{3} b\left(g_{\sigma} \sigma\right)^{3}-\frac{1}{4} c\left(g_{\sigma} \sigma\right)^{4}+\frac{1}{2}\left(\partial_{\mu} \delta \partial^{\mu} \delta-m_{\delta}^{2} \delta^{2}\right) \\
& +\frac{1}{2} m_{\omega}^{2} \omega_{\mu} \omega^{\mu}-\frac{1}{4} \omega_{\mu \nu} \omega^{\mu \nu}+\frac{1}{2} m_{\rho}^{2} \boldsymbol{\rho}_{\mu} \cdot \boldsymbol{\rho}^{\mu}-\frac{1}{4} \boldsymbol{\rho}_{\mu \nu} \cdot \boldsymbol{\rho}^{\mu \nu}
\end{aligned}
$$

where the antisymmetric tensors of vector mesons are given by

$$
\omega_{\mu \nu}=\partial_{\mu} \omega_{\nu}-\partial_{\nu} \omega_{\mu}, \quad \rho_{\mu \nu} \equiv \partial_{\mu} \boldsymbol{\rho}_{\nu}-\partial_{\nu} \boldsymbol{\rho}_{\mu} .
$$

In the $R M F$ approach all effective meson fields can be expressed via their mean values, simply related to baryon and scalar nucleon densities. In this way only nucleon degress of freedom are left to describe dynamics and thermodynamics of the system $[49,51]$. The nucleon chemical potential and effective mass in nuclear medium are

$$
\mu_{i}=\mu_{i}^{*}+g_{\omega} \omega+g_{\rho} \tau_{3 i} \rho
$$

$$
M_{i}^{*}=M-g_{\sigma} \sigma-g_{\delta} \tau_{3 i} \delta,
$$

where $M$ is the free nucleon mass, $\tau_{3 p}=1$ for proton and $\tau_{3 n}=-1$ for neutron, and $\mu_{i}^{*}$ is the effective chemical potential which reduces to Fermi energy $E_{F i}^{*}=\sqrt{k_{F}^{i^{2}}+M_{i}^{*^{2}}}$ at zero temperature. The baryon and isospin chemical potentials in the hadron phase are defined as

$$
\mu_{B}^{H}=\frac{\mu_{p}+\mu_{n}}{2}, \quad \mu_{3}^{H}=\frac{\mu_{p}-\mu_{n}}{2} .
$$

The energy density and pressure of nuclear matter at finite temperature can be derived as 


$$
\begin{aligned}
\varepsilon^{H} & =\sum_{i=p, n} \frac{2}{(2 \pi)^{3}} \int d^{3} \boldsymbol{k} \sqrt{k^{2}+M_{i}^{* 2}}\left(f_{i}(k)+\bar{f}_{i}(k)\right)+\frac{1}{2} m_{\sigma}^{2} \sigma^{2}+\frac{b}{3}\left(g_{\sigma} \sigma\right)^{3}+\frac{c}{4}\left(g_{\sigma} \sigma\right)^{4}+\frac{1}{2} m_{\delta}^{2} \delta^{2}+\frac{1}{2} m_{\omega}^{2} \omega^{2}+\frac{1}{2} m_{\rho}^{2} \rho^{2}, \\
P^{H} & =\sum_{i=p, n} \frac{1}{3} \frac{2}{(2 \pi)^{3}} \int d^{3} \boldsymbol{k} \frac{k^{2}}{\sqrt{k^{2}+M_{i}^{* 2}}}\left(f_{i}(k)+\bar{f}_{i}(k)\right)-\frac{1}{2} m_{\sigma}^{2} \sigma^{2}-\frac{b}{3}\left(g_{\sigma} \sigma\right)^{3}-\frac{c}{4}\left(g_{\sigma} \sigma\right)^{4}-\frac{1}{2} m_{\delta}^{2} \delta^{2}+\frac{1}{2} m_{\omega}^{2} \omega^{2}+\frac{1}{2} m_{\rho}^{2} \rho^{2} .
\end{aligned}
$$

where $f_{i}(k)$ and $\bar{f}_{i}(k)$ are the fermion and antifermion distribution functions for protons and neutrons $(i=$ $p, n)$ :

$$
\begin{aligned}
& f_{i}(k)=\frac{1}{1+\exp \left\{\left(E_{i}^{*}(k)-\mu_{i}^{*}\right) / T\right\}}, \\
& \bar{f}_{i}(k)=\frac{1}{1+\exp \left\{\left(E_{i}^{*}(k)+\mu_{i}^{*}\right) / T\right\}} .
\end{aligned}
$$

The effective chemical potentials $\mu_{i}^{*}$ are determined by the nucleon densities

$$
\rho_{i}=2 \int \frac{d^{3} \boldsymbol{k}}{(2 \pi)^{3}}\left(f_{i}(k)-\bar{f}_{i}(k)\right) .
$$

With the baryon density $\rho=\rho_{B}^{H}=\rho_{p}+\rho_{n}$ and isospin density $\rho_{3}^{H}=\rho_{p}-\rho_{n}$, the asymmetric parameter can be defined as

$$
\alpha^{H} \equiv-\frac{\rho_{3}^{H}}{\rho_{B}^{H}}=\frac{\rho_{p}-\rho_{n}}{\rho_{p}+\rho_{n}} .
$$

As model Lagrangians for the hadron phase, $N L \rho$ (the isovector scalar meson $\delta$ being not included) and $N L \rho \delta$ (with the $\delta$ meson) will be used. The effective meson couplings heve been chosen to reproduce good nuclear matter properties and even to represent a reasonable average of the density dependence predicted by Relativistic Dirac-Brueckner-Hartree-Fock calculation [52, 53], See details in Appendix A1 of Ref. [37, 38] and also Refs. $[48,49,51]$. A note about strangeness in the hadron phase can be found in Ref. [54].
For quark matter, we use the NJL model [57] to describe the interaction between quarks which is responsible for the quark dynamics at intermediate energies. The NJL model describes well the mesons spectra and successfully explains the dynamics of spontaneous breaking of chiral symmetry and its restoration at high densities / chemical potential [58-63]. The Lagrangian density in the three-flavor NJL model is taken as

$$
\begin{aligned}
\mathcal{L}_{q}= & \bar{q}\left(i \gamma^{\mu} \partial_{\mu}-\hat{m}_{0}\right) q+G \sum_{k=0}^{8}\left[\left(\bar{q} \lambda_{k} q\right)^{2}+\left(\bar{q} i \gamma_{5} \lambda_{k} q\right)^{2}\right] \\
& -K\left[\operatorname{det}_{f}\left(\bar{q}\left(1+\gamma_{5}\right) q\right)+\operatorname{det}_{f}\left(\bar{q}\left(1-\gamma_{5}\right) q\right)\right] .
\end{aligned}
$$

where $q$ denotes the quark fields with three flavors, $u, d$, and $s$, and three colors; $\hat{m}_{0}=\operatorname{diag}\left(m_{u}, m_{d}, m_{s}\right)$ in flavor space; $\lambda_{k}$ are the Gell-Mann matrices and $G$ and $K$ the four-point and six-point interacting constants, respectively. The four-point interaction term in the Lagrangian keeps the $S U_{V}(3) \times S U_{A}(3) \times U_{V}(1) \times U_{A}(1)$ symmetry, while the 't Hooft six-point interaction term breaks the $U_{A}(1)$ symmetry.

As an effective model, the NJL model is not renormalizeable, so a cut-off $\Lambda$ is implemented in 3-momentum space for divergent integration. We take the model parameters: $\Lambda=603.2 \mathrm{MeV}, G \Lambda^{2}=1.835, K \Lambda^{5}=12.36$, $m_{u, d}=5.5$ and $m_{s}=140.7 \mathrm{MeV}$, determined by Rehberg, Klevansky, and Hüfner in Ref. [64] by fitting $f_{\pi}, M_{\pi}, m_{K}$ and $m_{\eta}$ to their experimental values.

The thermodynamical potential in 3-flavor quark system in mean field approximation is

$$
\begin{aligned}
\Omega^{Q}= & -2 N_{c} \sum_{i=u, d, s} \int \frac{d^{3} \boldsymbol{k}}{(2 \pi)^{3}}\left\{\beta^{-1} \ln \left[1+e^{-\beta\left(E_{i}(k)-\mu_{i}\right)}\right]+\beta^{-1} \ln \left[1+e^{-\beta\left(E_{i}(k)+\mu_{i}\right)}\right]\right. \\
& \left.+E_{i}\right\}+2 G\left(\phi_{u}{ }^{2}+\phi_{d}{ }^{2}+\phi_{s}{ }^{2}\right)-4 K \phi_{u} \phi_{d} \phi_{s}+C
\end{aligned}
$$

where $C$ is a constant to be fixed by physics conditions, $N_{c}=3$ is the number of color degrees of freedom,
$E_{i}(k)=\sqrt{k^{2}+M_{i}^{* 2}}$ is energy-momentum dispersion relation of the quark flavor $i$, and $\mu_{i}$ is the corresponding 
chemical potential. The dynamical quark masses and quark condensates are coupled by the following equations:

$$
\begin{aligned}
& M_{i}^{*}=m_{i}-4 G \phi_{i}+2 K \phi_{j} \phi_{k} \quad(i \neq j \neq k), \\
& \phi_{i}=-2 N_{c} \int_{\Lambda} \frac{d^{3} \boldsymbol{k}}{(2 \pi)^{3}} \frac{M_{i}^{*}}{E_{i}}\left(1-n_{i}(k)-\bar{n}_{i}(k)\right),
\end{aligned}
$$

here $n_{i}(k)$ and $\bar{n}_{i}(k)$ are Fermi-Dirac distribution function of quark and antiquark

$$
n_{i}(k)=\frac{1}{1+\exp \left\{\left(E_{i}(k)-\mu_{i}\right) / T\right\}},
$$

and

$$
\bar{n}_{i}(k)=\frac{1}{1+\exp \left\{\left(E_{i}(k)+\mu_{i}\right) / T\right\}} .
$$

The pressure and energy density of the quark system are:

$$
\begin{aligned}
P^{Q}= & 2 N_{c} \sum_{i=u, d, s} \int \frac{d^{3} \boldsymbol{k}}{(2 \pi)^{3}} \frac{k^{2}}{3 E_{i}(k)}\left(n_{i}(k)+\bar{n}_{i}(k)\right)+2 N_{c} \sum_{i=u, d, s} \int_{\Lambda} \frac{d^{3} \boldsymbol{k}}{(2 \pi)^{3}} E_{i}(k)-2 G\left(\phi_{u}^{2}+\phi_{d}^{2}+\phi_{s}^{2}\right) \\
& +4 K \phi_{u} \phi_{d} \phi_{s}, \\
\varepsilon^{Q}= & 2 N_{c} \sum_{i=u, d, s} \int \frac{d^{3} \boldsymbol{k}}{(2 \pi)^{3}} E_{i}(k)\left(n_{i}(k)+\bar{n}_{i}(k)\right)-2 N_{c} \sum_{i=u, d, s} \int_{\Lambda} \frac{d^{3} \boldsymbol{k}}{(2 \pi)^{3}} E_{i}(k)+2 G\left(\phi_{u}{ }^{2}+\phi_{d}{ }^{2}+\phi_{s}{ }^{2}\right) \\
& -4 K \phi_{u} \phi_{d} \phi_{s} .
\end{aligned}
$$

If we define

$$
\begin{aligned}
B_{e f f}= & -2 N_{c} \sum_{i=u, d, s} \int_{\Lambda} \frac{d^{3} \boldsymbol{k}}{(2 \pi)^{3}} E_{i}(k) \\
& +2 G\left(\phi_{u}{ }^{2}+\phi_{d}{ }^{2}+\phi_{s}{ }^{2}\right)-4 K \phi_{u} \phi_{d} \phi_{s},
\end{aligned}
$$

we will find $B_{\text {eff }}$ acting exactly as an effective bag constant, just like the bag constant in the MIT-Bag model. The difference is that now $B_{\text {eff }}$ depends on the interaction between different quarks as well as on density and temperature. The same holds true for the Fermi motion contribution. For all the integrations the cut-off is implemented, together with the irrelevant thermodynamical constant $C$ of Eq.12, as usual by requiring the energy density and pressure equal to zero in the vacuum, ie., $\varepsilon^{Q}=P^{Q}=0$ at zero temperature and density.

The number density of quark flavor $i$ can be derived with the relation $\rho_{i}=-\partial \Omega_{q} / \partial \mu_{i}$

$$
\rho_{i}=2 N_{c} \int \frac{d^{3} \boldsymbol{k}}{(2 \pi)^{3}}\left(n_{i}(k)-\bar{n}_{i}(k)\right) .
$$

As we have mentioned in the Introduction, although the strangeness could be produced at finite temperature in heavy-ion collision, the number of net strange quark is zero before the hadronization takes place in the expanding process. So we must keep $\mu_{s}=0$ in the calculation according to Eq. (20), but the strange quarks in loop diagrams do contribute to the $u, d$ self energies via a sixpoint interaction, and then they can affect the dynamical mass of $u, d$ quark according to Eq. (13). The corresponding definitions of density and chemical potential in quark phase are as follows

$$
\begin{gathered}
\rho_{B}^{Q}=\frac{1}{3}\left(\rho_{u}+\rho_{d}\right), \quad \rho_{3}^{Q}=\rho_{u}-\rho_{d}, \\
\mu_{B}^{Q}=\frac{3}{2}\left(\mu_{u}+\mu_{d}\right), \quad \mu_{3}^{Q}=\frac{1}{2}\left(\mu_{u}-\mu_{d}\right) .
\end{gathered}
$$

The asymmetry parameter for quark phase is defined by

$$
\alpha^{Q} \equiv-\frac{\rho_{3}^{Q}}{\rho_{B}^{Q}}=3 \frac{\rho_{d}-\rho_{u}}{\rho_{u}+\rho_{d}} .
$$

The above introduction is a separate description of the hadronic and the quark phase. The goal of the present work is to extend these studies to the hadron-quark phase transition, in particular to stress the effect of dynamical quark masses.

Since there are two conserved quantity, baryon number and isospin, during the phase transition, the Gibbs criteria (thermal, chemical and mechanical equilibrium) should be implemented for the mixed phase. General discussion of phase transitions in multicomponent systems can be found in Ref.[29]. 
If we define $\chi$ the fraction of quark matter in the mixed phase, the Gibbs conditions for the mixed phase are

$$
\begin{aligned}
& \mu_{B}^{H}\left(\rho_{B}, \rho_{3}, T\right)=\mu_{B}^{Q}\left(\rho_{B}, \rho_{3}, T\right) \\
& \mu_{3}^{H}\left(\rho_{B}, \rho_{3}, T\right)=\mu_{3}^{Q}\left(\rho_{B}, \rho_{3}, T\right) \\
& P^{H}\left(\rho_{B}, \rho_{3}, T\right)=P^{Q}\left(\rho_{B}, \rho_{3}, T\right),
\end{aligned}
$$

where $\rho_{B}=(1-\chi) \rho_{B}^{H}+\chi \rho_{B}^{Q}$ is the total baryon density in the mixed phase, and the total isospin density is $\rho_{3}=(1-\chi) \rho_{3}^{H}+\chi \rho_{3}^{Q}$. With the initial condition of asymmetric parameter $\alpha^{H}$ in heavy-ion collision, the global asymmetry parameter $\alpha$ for the mixed phase should be

$$
\begin{aligned}
& \alpha \equiv-\frac{\rho_{3}}{\rho_{B}}=-\frac{(1-\chi) \rho_{3}^{H}+\chi \rho_{3}^{Q}}{(1-\chi) \rho_{B}^{H}+\chi \rho_{B}^{Q}}, \\
& \alpha^{H}(\chi=0)=\alpha^{Q}(\chi=1)
\end{aligned}
$$

according to the charge conservation.

\section{NUMERICAL RESULTS AND DISCUSSION}

Before presenting the phase diagram of hadron-quark phase transitions with the NJL quark model, we firstly display some results with the MIT-Bag model in which free fermions are considered with the current mass $m_{u}=$ $m_{d}=5.5 \mathrm{MeV}$. As for the bag constant, different values are used in literature, here we take $\left(B_{\text {MIT }}\right)^{1 / 4}=160 \mathrm{MeV}$ and $190 \mathrm{MeV}$, respectively, for comparison. All that will be useful for general comments on the nature of the phase transition as well as for a better understanding of the dynamical mass effects in the NJL approach.

We will pay more attention to the phase diagram of asymmetric matter. Indeed the onset density of the hadron-quark phase transition for asymmetric matter is smaller than in symmetric matter [36-40], then with the possibility to probe it in heavy-ion collision at the new planned facilities, for example FAIR at GSI-Darmstadt and NICA at JINR-Dubna, We focus the attention on experiments with neutron-rich stable heavy beams, where large intensities can be reached. Here the largest accessible isospin asymmetry parameter $\alpha$ is just above 0.2 (e.g. we have $\alpha=0.227$ in ${ }^{238} \mathrm{U}+{ }^{238} \mathrm{U}$ collision, see also the Table II in Ref.[39]). Therefore we will consider $\alpha=0.2$ in our calculation, just as taken in Refs. [37-40]. Of course the use of more asymmetric unstable beams will enhance the isospin effects described here.

\section{A. Some results with the MIT-Bag quark EoS}

In Figs. 1 and 2 we plot the $T-\rho_{B}$ and $T-\mu_{B}$ phase diagrams. In each figure the solid curves are the phasetransition lines from nuclear matter to quark matter for $\left(B_{\mathrm{MIT}}\right)^{1 / 4}=160 \mathrm{MeV}(190 \mathrm{MeV})$, and the corresponding dash-dot curves are the transition lines to pure quark matter. $N L \rho$ hadron $E o S$ has been used in the calculation.

From the two figures, it easy to see that the decrease of bag constant reduces the onset density of quark matter, and in general that the phase transition curve highly depends on the value of bag constant, which is one of the motivations of this paper to use the NJL model to investigate the phase transition. In the Fig. 2 we see a

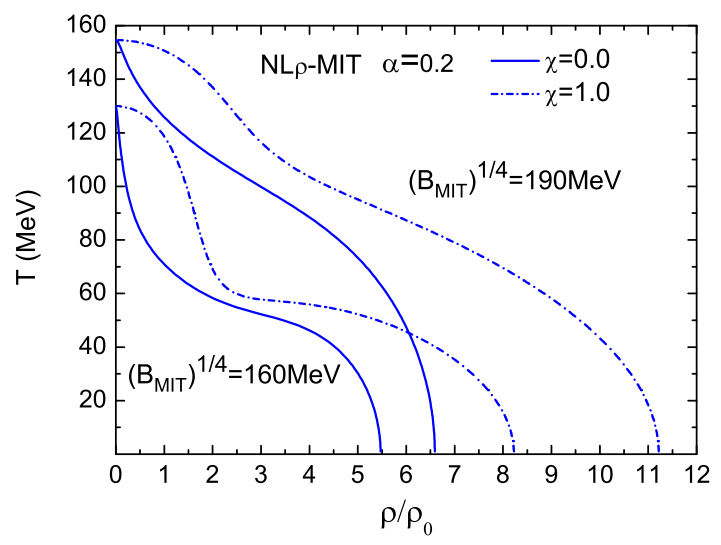

FIG. 1: (Color on line) The $T-\rho_{B}$ plane of asymmetric matter with the isospin ratio $\alpha=0.2$ for $\left(B_{\text {MIT }}\right)^{1 / 4}=160 \mathrm{MeV}$ and $190 \mathrm{MeV}$. The region between the solid and dash-dot curve gives the binodal surface of the mixed phase, where the hadron and quark matter coexist. $N L \rho$ parametrization is used for the hadron phase.

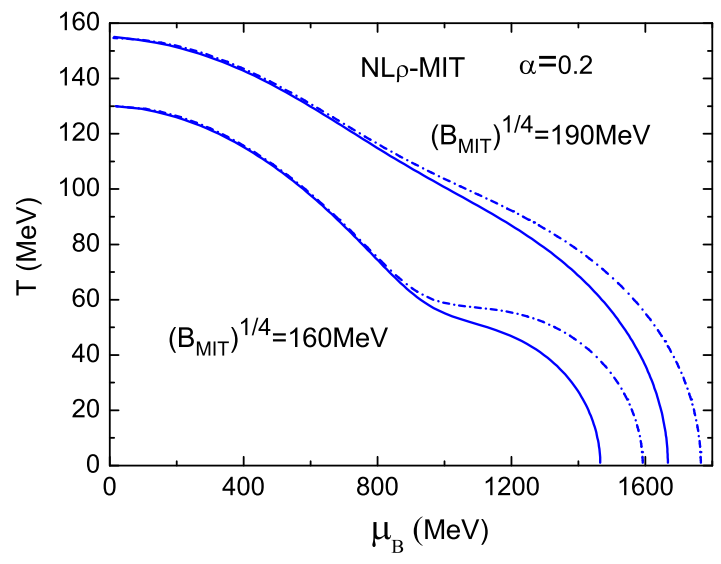

FIG. 2: (Color on line) The $T-\mu_{B}$ plane of asymmetric matter with the isospin ratio $\alpha=0.2$ for $\left(B_{\text {MIT }}\right)^{1 / 4}=160 \mathrm{MeV}$ and $190 \mathrm{MeV}$. The curves are like in the previous figure. $N L \rho$ parametrization is used for the hadron phase.

variation of the baryon chemical potential along the transition from pure hadron to pure quark phase, that could be interpreted as an evidence of a continuous second order phase transition for asymmetric matter, see [36]. We like to comment this point that we will see also clearly in the following results with the $N J L$ quark EoS. 
The transition is of first order, with the presence of a coexistence mixed phase, in both cases of symmetric and asymmetric matter. For symmetric matter, with only one conserved charge $\rho_{B}$, we have the expected discontinuities of thermodynamical quantities like entropy, pressure, at fixed chemical potential $\mu_{B}$. The presence of two conserved charges, $\rho_{B}$ and $\rho_{3}$, in asymmetric matter keeps the first order nature of the transition but changes some properties inside the mixed phase. Due to the presence of a new degree of freedom the interaction can choose the most energetically favored charge densities in each phase, at each relative concentration, in order to minimize the free energy of the system [29]. We can have an increase of pressure and chemical potential inside the mixed phase but the transition is still of first order since for each $\chi$ fraction we have a discontinuity in the $\rho_{B}$ and $\rho_{3}$ densities of the two phases in equilibrium. We note that this effect, directly related to the internal interaction in the two phases, leads to the important isospin distillation mechanism $\left(\alpha^{Q}>\alpha^{H}\right)$ inside the mixed phase, as shown later, of interest for possible experimental signals. Similar properties are present in the liquid-gas transition for dilute asymmetric nuclear matter, which is behind the multifragmentation processes at the Fermi energies $[50,51]$.

Before closing the discussion of MIT-Bag results we would like to add a few comments about the interpretation of the $T-\rho_{B}$ and $T-\mu_{B}$ phase diagrams of Figs. 1, 2.

For the lower bag costant value $\left(B^{1 / 4}=160 \mathrm{MeV}\right)$ we clearly see a squeezing of the binodal surface (mixed phase region) in the $T-\rho_{B}$ plane at temperature $T \simeq$ $60 \mathrm{MeV}$, corresponding to a baryon chemical potential $\mu_{B} \simeq 900 \mathrm{MeV}$, and a re-opening at higher temperature and smaller density (or chemical potential). The same is happening for the large bag constant, although less evident.

We note that this effect is appearing just around a transition chemical potential equal to the nucleon effective mass $\mu_{B} \simeq M_{n, p}^{*}$. We have a simple interpretation:

- The "opening" at low baryon density and high temperature.

Here the transition $\mu_{B}$ is below the nucleon effective mass (not much reduced at low densities) and the effective chemical potential $\mu_{i}^{*}$ even smaller. Thus in the hadron part $E_{F, i}^{*}>\mu_{i}^{*}$ and the fermion distribution function of the nucleons will be rather small. Therefore $\rho_{B}^{H}$, Eq. (9), will show a slow increase with $\mu_{B}$, while the pressure, mostly of thermal nature, is increasing (even for the positive antifermion contribution). At variance in the quark sector $\mu_{B}$ is always much larger than the used current quark masses $(5.5 \mathrm{MeV})$ and so we see a fast increase of $\rho_{B}^{Q}$ to balance the hadron pressure.

- The "re-opening" at high baryon density and low temperature.
With the increasing of baryon density/chemical potential, the nucleon effective mass decreases and the transition $\mu_{i}<M_{i}^{*}$ to $\mu_{i}>M_{i}^{*}$ takes place. Thus the fermion distribution function of nucleons and $\rho_{B}^{H}$ will show a fast increase with $\mu_{B}$. The interaction part of the hadron pressure will also increase. In correspondence we will need a fast increase even of the $\rho_{B}^{Q}$ in order to keep the $P^{H}=P^{Q}$ balance.

We remark that the first argument is not anymore valid in the NJL frame, since the quark masses at low density and finite temperature recover the much larger constituent quark values. Therefore we would expect important qualitative differences in this low $\mu_{B}$ phase diagram region when we take into account the chiral mass dynamics, see Subsection C.

\section{B. Results with the $N J L$ quark $E o S$}

In Figs. 3 and Fig. 4 we present the $P-\rho_{B}$ phase diagram, within the $N L \rho-N J L$ two- $E o S$ scheme, respectively for symmetric and asymmetric $(\alpha=0.2)$ matter. For each temperature the mixed phase region is between the two solid dots.

Clearly the pressure is a constant in the mixed phase of symmetric matter, Fig. 3, just the same as with Maxwell construction. At variance in the asymmetric case, Fig. 4, we have a monotonous increase. It is interesting to note that pressure rising is faster in the first part of the mixed phase (more evident for the lower temperatures $\mathrm{T}=30,60$ $\mathrm{MeV})$. This is due to the isospin distillation effect, i.e. a large $\rho_{d}-\rho_{u}$ asymmetry for reduced quark fractions, see next Subsection, which is increasing the quark pressure as we can expect from Eq. (17).

From Figs. 3, 4, we can also see that the size of the mixed phase shrinks with temperature. Meanwhile the onset density becomes smaller, opening the possibility of probing the coexistence phase in heavy-ion collision at intermediate energies. This effect is further enhanced by the isospin asymmetry as we will discuss in the next Subsection.

\section{Symmetry energy effects}

In order to study symmetry energy effects we have performed the hadron-quark transition calculations with the hadron EoSs $N L \rho$ and $N L \rho \delta$ which present rather different symmetry terms at high baryon density, stiffer when the $\delta$ meson is included $[49,51]$.

In Fig. 5 we plot the (identical) $T-\rho_{B}$ phase diagrams of symmetric matter. For asymmetric matter $\alpha=0.2$ in Fig. 6 we show the results for the parameter set NL $\rho$ and in Fig. 7 for the parameter set $N L \rho \delta$. Now the mixed phase region, the binodal surface, is rather different. In general the onset density of the mixed phase in asymmetric matter is smaller than that in symmetric matter, simi- 


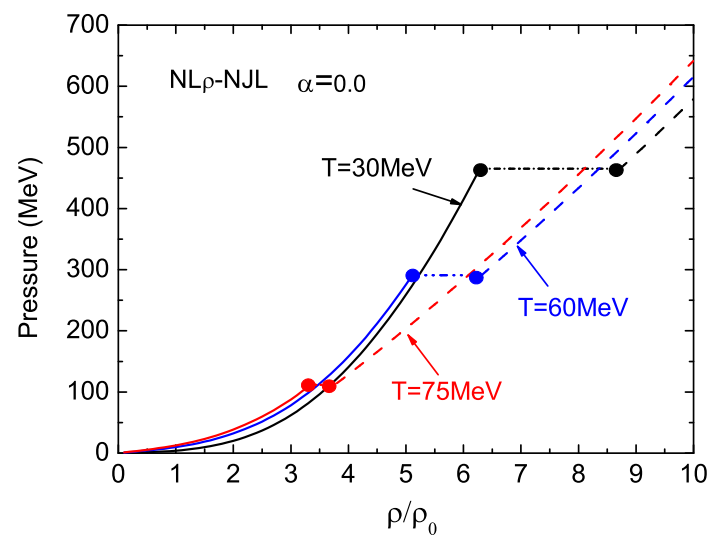

FIG. 3: (Color on line) Pressure of symmetric matter as a function of baryon number density at different temperatures. The solid dots correspond to the limits of the mixed phase. $N L \rho-N J L$ two-EoS calculation.

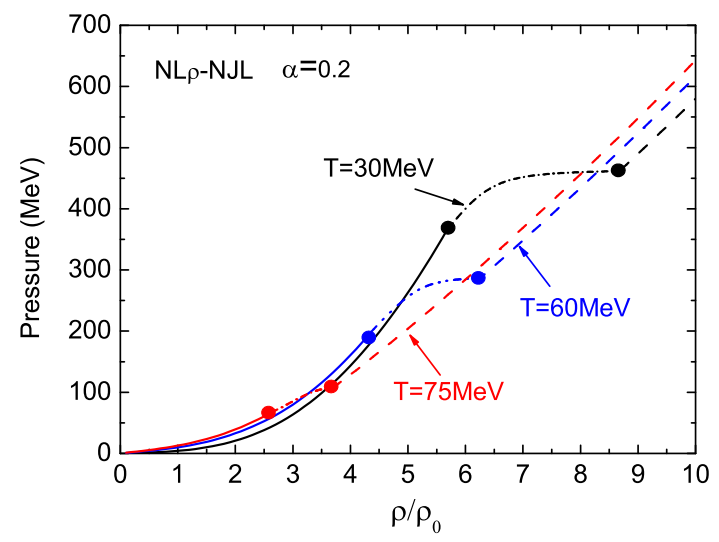

FIG. 4: (Color on line) Like previous figure, but for the asymmetric matter $\alpha=0.2$ case.

lar to the results obtained by the MIT-Bag model [36-40]. In fact the mechanism is the same: for a given baryon density in isospin asymmetric matter we have a larger repulsion in the hadron phase since the symmetry term is less important in the quark sector for both MIT-Bag and NJL effective lagrangians.

If the $N L \rho \delta$ parameter set is used, the onset density will be further reduced, as shown in Fig. 7. This is nicely due to the fact that at high baryon density the symmetry energy of hadron matter with the parameter set $N L \rho \delta$ is much larger than in the $N L \rho$ case, see the following. All that also indicates that isospin effects can be very important for hadron-quark phase transition in heavyion collision in order to shed light on nuclear interactions in an hot and dense medium.

In Figs. 5, 6 and 7 the curves corresponding to a given quark fraction $\chi$ inside the mixed phase are also shown. We note that the isospin effects are mainly relevant in the initial part of the coexistence region. This is consistent with the interpretation of the pressure behavior in Fig. 4. We finally remark that such lower density zone can be reached even at relatively lower beam energies.

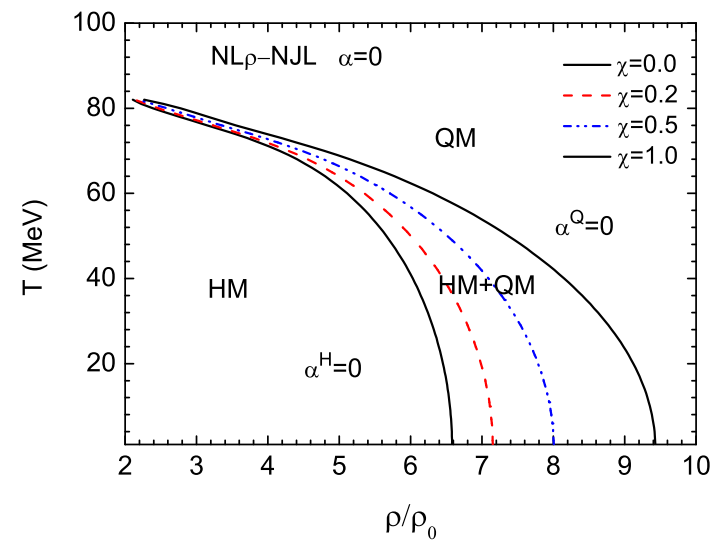

FIG. 5: (Color on line) Phase diagram in the $T-\rho_{B}$ plane for symmetric matter with the parameter set $N L \rho$. Of course the same curves are obtained with the parameter set $N L \rho \delta$.

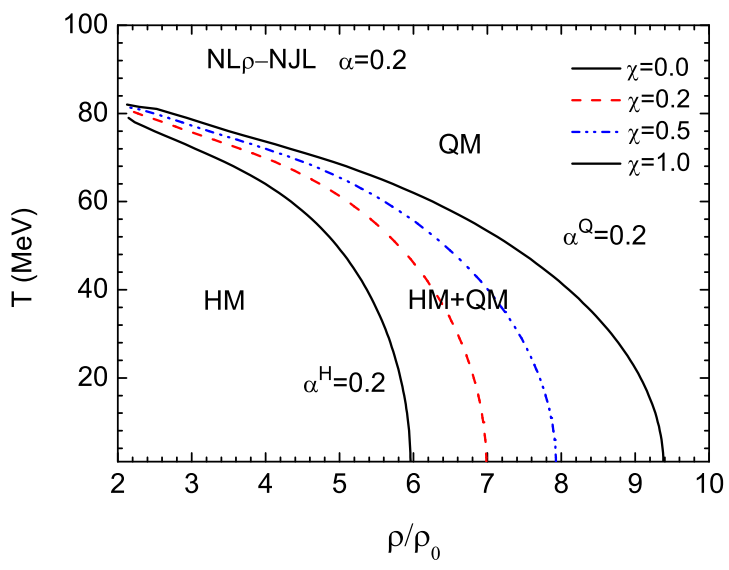

FIG. 6: (Color on line) Phase diagram in the $T-\rho_{B}$ plane for asymmetric matter with the parameter set $N L \rho$.

To understand the role of the symmetry term on the hadron-quark phase transition, we further investigate the symmetry energy $E_{\text {sym }}$ in the two phases, defined in general by $[37,40]$

$$
(E / A)_{\alpha_{i}}=(E / A)_{\alpha_{i}=0}+E_{\text {sym }} \alpha_{i}^{2}
$$

where $\alpha_{i}=\alpha^{H}$ for hadron matter, $\alpha^{Q}$ for quark matter.

From the hadron (RMF approach) and the quark (MIT-Bag, NJL) EoSs described before we can evaluate the symmetry energy as a function of the baryon density at fixed temperature. We display the result in Fig. 8 for both hadron and quark matter at $\mathrm{T}=0,80 \mathrm{MeV}$. It is clear the symmetry energy of quark matter, only due to the Fermi kinetic term in both MIT and NJL schemes, is 


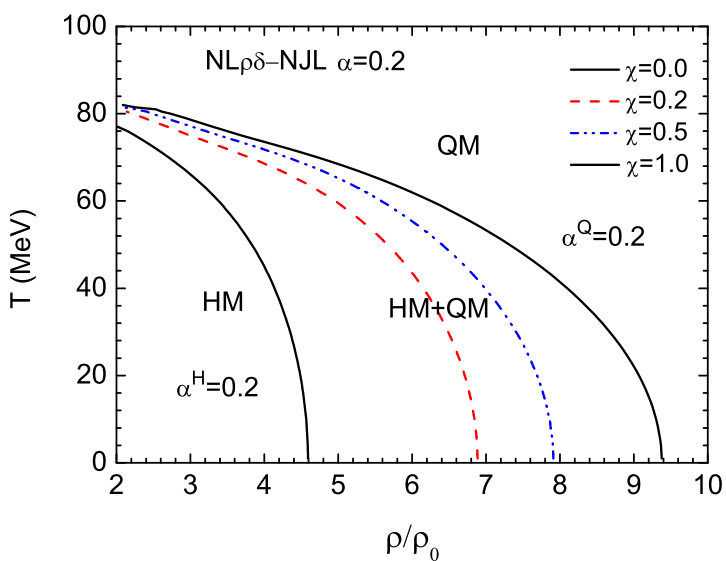

FIG. 7: (Color on line) Phase diagram in the $T-\rho_{B}$ plane for asymmetric matter with the parameter set $N L \rho \delta$.

much smaller than that of hadron matter, where also interaction isovector mesons contribute. Moreover in general we get a decrease of the symmetry energy at lower densities and higher temperatures due to a smaller contribution from the Fermi kinetic motion.

The symmetry energy of hadron matter with the parameter set $N L \rho \delta$ is larger than that of the $N L \rho$ case. As discussed before by comparing Fig. 6 and Fig. 7, this leads to a smaller onset density of the transition. It is interesting to note that this is a genuine relativistic effects since the scalar covariant nature of the isovector $\delta$ meson contributes to increase the symmetry energy at high densities directly with a larger repulsion and indirectly via a splitting of the neutron/proton effective masses, with $M_{n}^{*}<M_{p}^{*}$, see details in refs. $[49,51]$.

We see that larger is the symmetry energy difference between hadron and quark phase, smaller is the onset density of the phase transition. Moreover a big variation of the symmetry term will strengthen the observable signals of phase transition, which will be discussed later. Of course all these effects will be enhanced by the global isospin asymmetry of the system, suggesting the interest in experiments with very neutron-rich unstable beams.

In Fig. 9 we plot the $T-\mu_{B}$ phase diagram for symmetric and asymmetric matter with both the parameter sets $N L \rho$ and $N L \rho \delta$. For the symmetric matter at a given temperature, $\mu_{B}$ is constant in the mixed phase, i.e. varying the quark matter fraction $\chi$, then we have only one transition line (the empty circles). At variance, like in the MIT-Bag calculation of Fig. 2, in the asymmetric case we have a monotonous $\mu_{B}$ increase with increasing $\chi$, so there are two curves in $T-\mu_{B}$ plane presenting the start and end, respectively, of the transition from nuclear to quark matter. What is important is that the chemical potentials of the onset of the transition $(\chi=0)$ in asymmetric matter are always smaller than the corresponding ones of symmetric matter. This is more evident with the parameter set $N L \rho \delta$ (dotted curve), which again shows

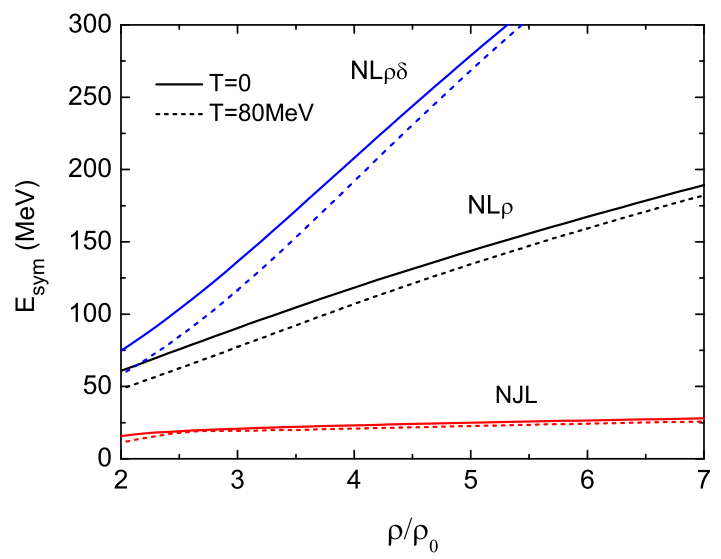

FIG. 8: (Color on line) Symmetry energy of nuclear matter with the parameter sets $N L \rho, N L \rho \delta$ and that of $N J L$ quark matter. Temperature $T=0,80 \mathrm{MeV}$.

the importance of symmetry energy effects.

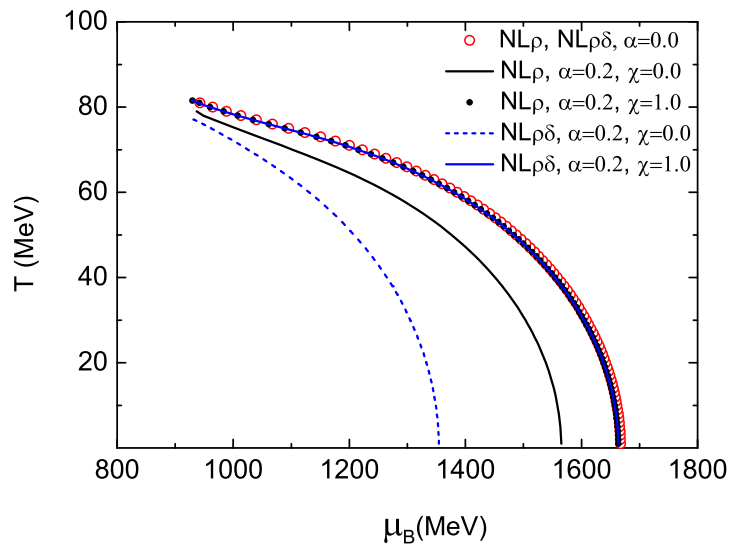

FIG. 9: (Color on line) Phase diagram in the $T-\mu_{B}$ plane for symmetric and asymmetric matter with the parameter sets $N L \rho$ and $N L \rho \delta$. Always $N J L$ quark $E o S$.

\section{Isospin Distillation inside the mixed phase}

In Figs. 10 and 11, we show the variation of the isospin asymmetry parameters of hadron $\left(\alpha^{H}\right)$ and quark $\left(\alpha^{Q}\right)$ matter inside the mixed phase at various temperatures, with the global asymmetry $\alpha=0.2$. We clearly see the much larger values of $\alpha^{Q}$ when the quark phase starts forming, roughly for a $\chi$ fraction between 0.0 and 0.4 . Of course the effect disappears when the pure quark phase is reached $(\chi=1)$ where the global asymmetry is recovered just for the two charges $\left(\rho_{b}, \rho_{3}\right)$ conservation. This is a nice Isospin Distillation effect ruled by the symmetry energy gap in the two phases, as confirmed by the enhancement in Fig. 11, where the more symmetry repulsive $N L \rho \delta E O S$ is used for the hadron part. 
We note that the isospin asymmetry of quark matter decreases with the enhancement of temperature. This is due to a general decrease of symmetry energy effects at higher temperatures (and lower densities), as we can clearly see in Fig. 9.

The color pairing interaction at high density can reduce the isospin distillation since it is energetically equivalent to the introduction of an effective symmetry repulsion in the quark phase $[38,40]$. However the isospin effects discussed before are still present. Moreover we can expect the pairing correlations to be less important with increasing temperature. Experiments focused to observe isospin effects in the mixed phase, using neutronrich heavy ion collisions at intermediate energies, appear very appealing.

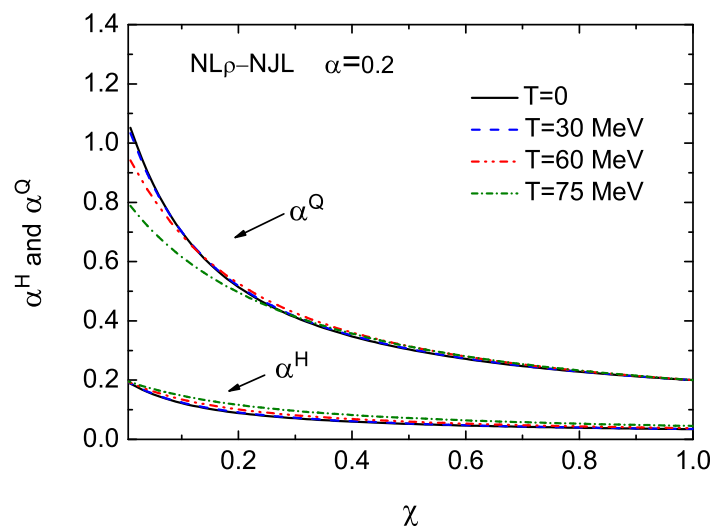

FIG. 10: (Color on line) Asymmetry of quark matter and nuclear matter inside the mixed phase at different temperatures. The parameter set $N L \rho$ is used for the hadron phase.

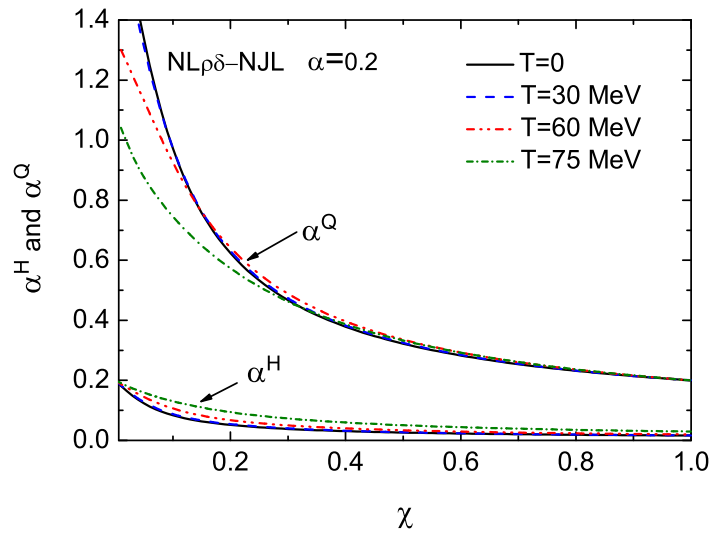

FIG. 11: (Color on line) Like in the previous Figure, but with the $N L \rho \delta E o S$ for the hadron phase.

This behavior of the quark isospin asymmetry inside the mixed phase of the hadron-phase transition will affect the following hadronization in the expansion stage, finally producing some observable signals in heavy-ion experiments. As suggested before in Ref. [37, 38], an inversion in the trend of the emission of neutron rich clusters, $\pi^{-} / \pi^{+}, K^{0} / K^{+}$yield ratios in high density regions, and an enhancement of the production of isospin-rich resonances and subsequent decays may be found. Besides, there is a controversial point of view about the enhancement of the yield ratio $\bar{\Lambda} / \bar{p}[65-69]$.

For instance, in [37] the reaction ${ }^{238} \mathrm{U}+{ }^{238} \mathrm{U}$ ( isospin asymmetry $\alpha=0.227$ ) at $1 A \mathrm{GeV}$ has been investigated in the RMF approach, and a rather exotic nuclear matter is formed with baryon density around $3-4 \rho_{0}$, temperature $50-60 \mathrm{MeV}$, likely inside the estimated mixed phase region, especially with the parameter set $N L \rho \delta$ for the hadron sector.

\section{New effect of the dynamical quark masses: a Critical-End-Point?}

If we compare MIT-Bag (with fixed current quark masses) and NJL (with chiral restoration mechanism) results for the mixed phase we remark only one main difference. As shown in Figs. 1, 2, phase diagrams in $T-\rho_{B}$ and $T-\mu_{B}$ planes are derived, using the MIT Bag model, with a critical temperature reached only at zero baryon density. At variance the binodal curves at high temperature and low density cannot be obtained with the NJL model and we see a narrowing of the coexistence region up to a kind of Critical - End - Point, Figs. 5, 6, 7 and 9. This important result derives from two qualitative new features of the NJL effective theory: i) the quark masses variation due to the chiral restoration, ii) the dependence of the effective Bag-constant $B_{\text {eff }}$, Eq. (19), on temperature and baryon density.

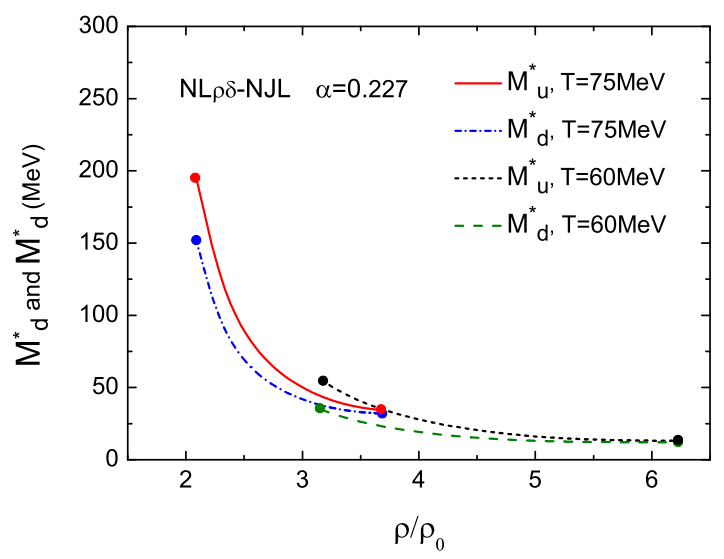

FIG. 12: (Color on line) Dynamical masses of $u, d$ quarks inside the mixed phase at different temperatures. The solid circles indicate the two density limits of the coexistence region. Asymmetric matter with $\alpha=0.227$. The $N L \rho \delta E o S$ is used for hadron phase.

The two effects are jointly leading to a determination of an End-Point of the mixed phase: 


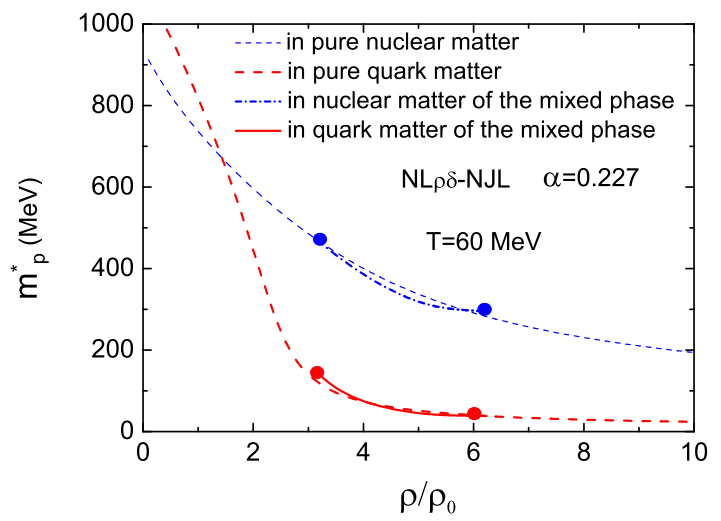

FIG. 13: (Color on line) Effective proton masses in the hadron (upper) and quark (lower) matter inside the mixed phase at $T=60 \mathrm{MeV}$. The solid circles indicate the two density limits of the coexistence region. Asymmetric matter with $\alpha=0.227$. The $N L \rho \delta E o S$ is used for hadron phase.

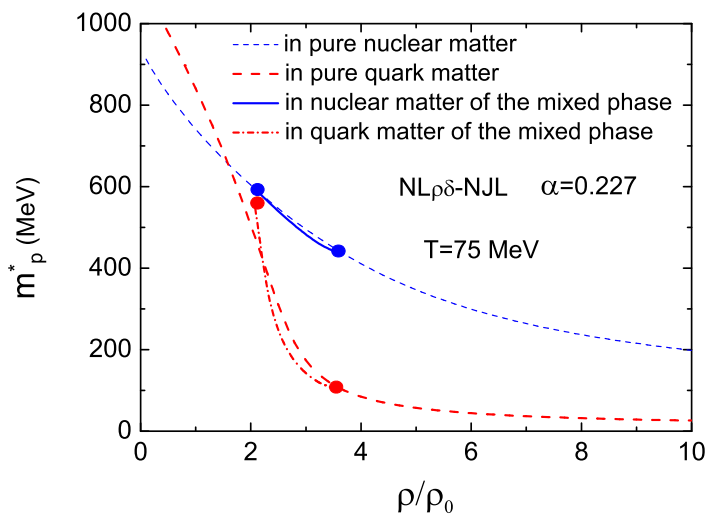

FIG. 14: (Color on line) Like in the previous Figure, but at temperature $\mathrm{T}=75 \mathrm{MeV}$.

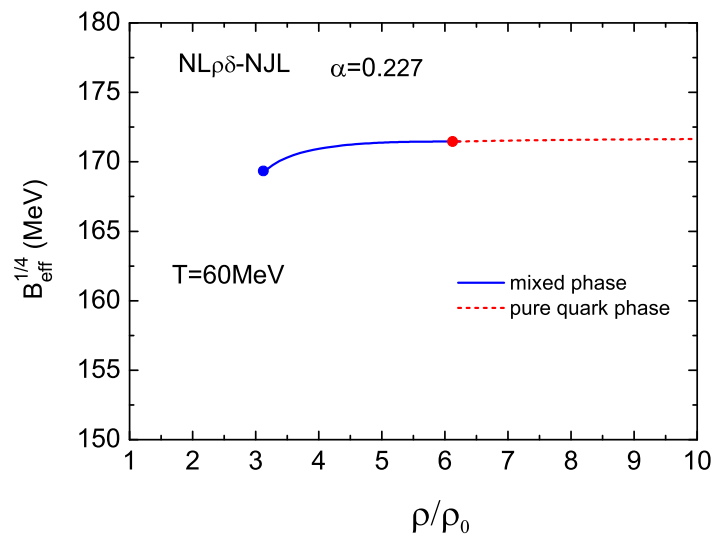

FIG. 15: (Color on line) Baryon density dependence of the NJL Effective Bag "constant", $\left(B_{\text {eff }}\right)^{1 / 4}$, at $T=60 \mathrm{MeV}$. The line ending with solid circles indicates coexistence region. Asymmetric matter with $\alpha=0.227$. The $N L \rho \delta E o S$ is used for hadron phase.

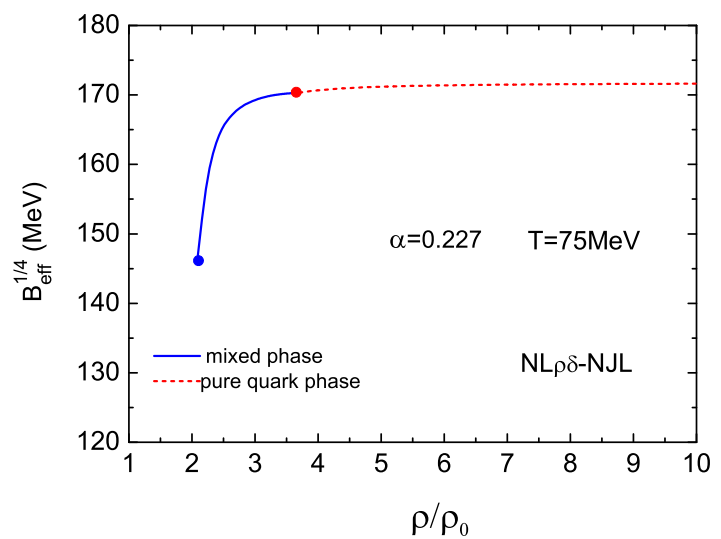

FIG. 16: (Color on line) Like in the previous Figure, but at temperature $T=75 \mathrm{MeV}$.

- The chiral dynamics largely increases the quark masses at low densities and finite temperatures, see Fig. 12 where we plot the $u, d$ effective masses inside the mixed phase at different temperatures, for asymmetric matter. If we reach the limit of a nucleon effective mass in the hadron phase smaller than the corresponding combination of quark effective masses, e.g. for protons $M_{p}^{*}<2 M_{u}^{*}+M_{d}^{*}$, we would have unphysical solutions for the Gibbs conditions since from Eqs. (9), (20) we will get only $\rho_{B}^{Q}<\rho_{B}^{H}$ results. This is confirmed by the Figs. 13, 14 , where we plot the proton effective mass in the hadron phase (solid line) and in the quark phase (dot-dashed line) inside the coexistence region at $T=60 \mathrm{MeV}$ and $T=75 \mathrm{MeV}$. We see that at the higher temperature we are close to a crossing, indication of the lack of physical solution at higher temperatures, as already seen in the corresponding $N L \rho \delta$ results of the previous Figs. 7 and 9 .

We have a final interesting comment about the quark mass splitting shown in Fig. 12 for asymmetric matter. The difference in the quark masses, with $M_{d}^{*}<M_{u}^{*}$, is larger at the onset of the mixed phase, where the isospin distillation effects induces a larger difference in the two quark-antiquark condensates.

- Actually we clearly see that in the same $(T, \rho)$ region we do not have solutions of the Gibbs conditions. This is due to the second qualitative new feature of the $N J L$ approach, the density and temperature dependence of the "Effective Bag Constant", Eq. 19, also related to the dynamical quark mass variation. As a consequence at low densities and high temperatures, for small values of the Bag constant we cannot get mixed phase solutions since the hadron pressure (mostly thermal) cannot equilibrate the quark pressure in the coexistence zone. In Figs. 15, 16 we show the density dependence 
of $\left(B_{\text {eff }}\right)^{1 / 4}$ at $T=60$ and $T=75 \mathrm{MeV}$ (the solid circles give the mixed phase limits). For the $T=75 \mathrm{MeV}$ case we see a sudden drop around the onset of the mixed phase, good indication of a lack of solution for larger temperatures.

In conclusion the chiral dynamics seems to lead to a Critical-End-Point $(C E P)$ of the first order hadronquark transition, around $T \simeq 80 \mathrm{MeV}$ and $\rho_{B} \simeq 2 \rho_{0}$ or $\mu_{B} \simeq 900 \mathrm{MeV}$. Beyond this point, i.e. at higher temperatures and smaller baryon densities, we have to follow an approach with just one effective $E o S$ able to describe both phases in order to check if we get a continous transition.

It is interesting to note that the $C E P$ appears when the NJL quark masses have reached the constituent quark values. However we are aware that the NJL approach, with only chiral dynamics, is not good at low densities and finite temperatures since confinement is not accounted for (in fact our $B_{\text {eff }}$ goes to zero), just where we can expect that hadron degrees of freedom will start to play a role [63]. A further investigation including a confinement mechanism is certainly required in order to confirm the End-Point evaluation and to understand the related physics. Finally correlation effects, beyond the mean field approximation, would be also more important.

In any case the fact that in the $\left(T, \mu_{B}\right)$ plane around the End Point we definitely get a derivative $d T / d \mu \neq 0$ (see Fig. 9) could be an indication that we are actually reaching a continous transition for chemical potentials and entropies, as suggested by the Clausius-Clapeyron Equation [38].

Finally two more remarks:

- From Figs. 5, 6, 7 and 9, we see that the EndPoint of the mixed phase is not depending on the global asymmetry of the system and on the symmetry term of the used hadron interaction. This is consistent with the reduced effect of the symmetry energy at high temperatures and low densities, Fig. 8.

- Isospin effects appear still relevant in the region just below the End-Point, $\mathrm{T} \subset(50-80) \mathrm{MeV}$ and $\rho_{B} \subset(2-4) \rho_{0}$, accessible in the transient compression stage of Heavy Ion Collisions at intermediate energies.

The latter point is interesting even because in that phase diagram zone the NJL and MIT-Bag results are very similar, for similar bag constants $B_{M I T} \simeq B_{\text {eff }}$ [38]. This makes more reliable the observed isospin effects.

\section{SUMMARY}

In this work, we investigate the hadron-quark phase transition in isospin asymmetric matter. We use a Two-
EoS approach as in all previous calculations. The novelty of this study is to insert chiral restoration effects on the quark masses using the 3-flavor NJL model for interacting quark instead of the MIT Bag model. We obtain the binodal surface of a first order hadron-quark phase transition in the region of $\rho_{B}>2 \rho_{0}$ and temperature $T$ less than about $80 \mathrm{MeV}$, available in heavy-ion experiments in the near future.

The calculated results show that the onset density of the phase transition is lowered with the increasing asymmetry parameter $\alpha$, property possible to be probed in the new planned facilities, for example, FAIR at GSIDarmstadt and NICA at JINR-Dubna, with realistic asymmetries for stable beams, see [37].

The phase diagrams at high density are like the ones given by the MIT Bag, model with appropriate bag constant, since the chiral symmetry is restored and the quark masses approach the current values. But the dynamical quark mass becomes more and more important with the reduction of baryon number density and the increase of temperature, causing a reduction of the Effective Bag Constant. The resulting effect is more relevant just in the region $\mathrm{T} \subset(50-80) \mathrm{MeV}$ and $\rho_{B} \subset(2-4) \rho_{0}$, available with the new planed facilities, where some suggested important observables may be found as the signals of hadron-quark phase transition in the future.

The most interesting effect of the use of a consistent chiral quark mass dynamics is the appearance of a kind of Critical-End Point for the first order transition, around $T \simeq 80 \mathrm{MeV}$ and $\rho_{B} \simeq 2 \rho_{0}$ or $\mu_{B} \simeq 900 \mathrm{MeV}$. Furthermore the obtained phase diagram exhibits a region with confinement but chiral restored symmetry, as expected for the Quarkyonic matter [70].

At variance with the results with the MIT-Bag quark $E o S$, the phase diagram at lower density and higher temperature cannot be derived from the Gibbs conditions in a two-EOS model, which means that chiral symmetry is very important in the phase transition. This conclusion stimulates new efforts in the search of an unique $E o S$, with chiral dynamics and confinement mechanism, able to describe both phases in the region just above the suggested Critical-End-Point.

In any case reliable results have been obtained in the interesting region $\mathrm{T} \subset(50-80) \mathrm{MeV}$ and $\rho_{B} \subset(2-4) \rho_{0}$, that will undergo the test of experiments in a near future.

\section{Acknowledgments}

This work was supported in part by the National Natural Science Foundation of China under Grants Nos. 10875160, 11075037, 10935001 and the Major State Basic Research Development Program under Contract No. G2007CB815000. 
[1] F. R. Brown, et al, Phys. Rev. Lett. 65, 2491 (1990).

[2] M. Stephanov, K. Rajagopal, and E. Shuryak, Phys. Rev. Lett. 81, 4816 (1998).

[3] M. A. Halasz, A. D. Jackson, R. E. Shrock, M. A. Stephanov, and J. J. M. Verbaarschot, Phys. Rev. D 58, 096007 (1998).

[4] Z. Fodor and S. D. Katz, JHEP 03, 014 (2002); JHEP 04, 050 (2002); Phys. Lett. B 534, 87 (2002)..

[5] D. Toublan and J. B. Kogut, Phys. Lett. B 564, 212 (2003). 479 (2005).

[6] S. B. Rüster, V. Werth, M. Buballa, I. A. Shovkovy, and D. H. Rischke, Phys. Rev. D 72, 034004 (2005).

[7] Hiroaki Abuki, and Teiji Kunihiro, Nucl. Phys. A 768, 118 (2006).

[8] N. Kawamoto, K. Miura, A. Ohnishi, and T. Ohnuma, Phys. Rev. D 75, 014502 (2007).

[9] S. Rößner, C. Ratti, and W. Weise, Phys. Rev. D 75, 034007 (2007).

[10] A. Andronic, et al., Nucl. Phys. A 837, 65 (2010).

[11] P. Braun-Munzinger and J. Wambach, Rev. Mod. Phys. 81, 1031 (2009).

[12] M. Buballa, A.G. Grunfeld, A. E. Radzhabov and D. Scheffler, Prog. Part. Nucl. Phys. 62, 365 (2009).

[13] B-J. Schaefer, M. Wagner, J. Wambach, Phys. Rev. D 81, 074013 (2010).

[14] T. K. Herbst, J. M. Pawlowski, B-J. Schaefer, Phys. Lett. B 696, 58 (2011)

[15] K. Fukushima, T. Hatsuda, Rept. Prog. Phys. 74014001 (2011).

[16] I. Shovkovy, and Mei Huang, Nucl. Phys. B 564, 205 (2003).

[17] M. Huang and I. Shovkovy, Nucl. Phys. A 729, 835 (2003).

[18] M. Alford, A. Schmit, K. Rajagopal, and T. Schäfer, Rev. Mod. Phys. 80, 1455 (2008), and refs. therein..

[19] K. Fukushima, Nucl. Phys. B 591, 277 (2004); Phys. Rev. D 77, 114028 (2008).

[20] K. Kashiwa, H. Kouno , M. Matsuzaki , and M. Yahiro, Nucl. Phys. B 662, 26 (2008).

[21] H. Abuki, R. Anglani, R. Gatto, G. Nardulli and M. Ruggieri, Phys. Rev. D 78, 034034 (2008).

[22] W. J. Fu, Z. Zhang, Y. X. Liu, Phys. Rev. D 77, 014006 (2008).

[23] M. A. Stephanov, PoS LAT 2006, 024, 11 (2006). arXiv.hep-lat/0701002.

[24] S. Lawley, W. Bentz, and A. W. Thomas, Phys. Lett. B 632, 495 (2006).

[25] A. H. Rezaeian and H.-J. Pirner, Nucl. Phys. A 769, 35 (2006).

[26] S. Lawley, W. Bentz, and A.W. Thomas, J. Phys. G 32, 667 (2006).

[27] V. A. Dexheimer and S. Schramm, Phys. Rev. C 81, 045201 (2010).

[28] J. Steinheimer, S. Schramm, H. Stöcker, J. Phys. G 38, 035001 (2011).

[29] N. K. Glendenning, Phys. Rev. D 46, 1274 (1992).

[30] N. K. Glendenning and J. Schaffner-Bielich, Phys. Rev. Lett. 81, 4564 (1998); Phys. Rev. C 60, 025803 (1999).

[31] G. F. Burgio, M. Baldo, P. K. Sahu, and H.-J. Schulze, Phys. Rev. C 66, 025802 (2002).

[32] T. Maruyama, S. Chiba, H-J. Schulze, and T. Tatsumi,
Phys. Rev. D 76, 123015 (2007).

[33] F. Yang and H. Shen, Phys. Rev. C 77, 025801 (2008).

[34] G. Y. Shao and Y. X. Liu, Phys. Rev. C 82, 055801 (2010).

[35] J. Xu, L. W. Chen, C. M Ko, and B. A. Li, Phys. Rev. C 81, 055803 (2010).

[36] H. Müller, Nucl. Phys. A 618, 349 (1997).

[37] M. Di Toro, A. Drago, T. Gaitanos, V. Greco, and A. Lavagno, Nucl. Phys. A 775, 102 (2006).

[38] M. Di Toro et al., arXiv:0909.3247v3, Phys. Rev. C 83, 014911 (2011).

[39] R. Cavagnoli, C. Providência, and D. P. Menezes, arXiv:1009.3596v1.

[40] G. Pagliara and J. Schaffner-Bielich, Phys. Rev. D 81, 094024 (2010).

[41] C. Greiner, P. Koch, and H. Stoecker, Phys. Rev. Lett. 58, 1825 (1987).

[42] D. K. Srivastava and B. Sinha, Phys. Rev. Lett. 73, 2421 (1995).

[43] T. S. Biró, P. Lévai, J. Zimányi, Phys. Lett. B 347, 6 (1995).

[44] R. C. Hwa and C. B. Yang, Phys. Rev. C 67, 034902 (2003).

[45] R. J. Fries, B. Muller, and C. Nonaka, Phys. Rev. Lett. 90, 202303 (2003).

[46] V. Greco, C. M. Ko, and P. Lévai, Phys. Rev. Lett. 90, 202302 (2003).

[47] R. J. Fries, V. Greco, P. Sorensen, Ann. Rev. Nucl. Part. Sci. 58, 177 (2008).

[48] M. Di Toro et al., Progr. Part. Nucl. Phys. 62, 389 (2009).

[49] B. Liu, V. Greco, V. Baran, M. Colonna, M. Di Toro, Phys. Rev. C 65045201 (2002).

[50] Ph.Chomaz, M.Colonna, J.Randrup. Phys. Rep. 389, 263 (2004).

[51] V. Baran, M. Colonna, V. Greco, M. Di Toro, Phys. Rep. 410, 335205 (2005).

[52] F. Hofmann, C. M. Keil, H. Lenske, Phys. Rev. C 64 034314 (2001).

[53] P. Goegelein, E. N. E. van Dalen, C. Fuchs, H. Müther, Phys. Rev. C 77025802 (2008).

[54] Strangeness is not considered in the hadron EoS. This is due to two main reasons: i) Strange particle production is very limited at the intermediate energies of interest here; ii) From fully dynamical calculations of strange particle production it appears that in the short time interval of the transient high density state the strangeness chemical equilibrium is not reached $[55,56]$.

[55] G. Ferini, M. Colonna, T. Gaitanos, M.Di Toro, Nucl. Phys. A 762, 147 (2005).

[56] G. Ferini, T. Gaitanos, M. Colonna, M.Di Toro, H.H. Wolter, Phys. Rev. Lett. 97202301 (2006).

[57] Y. Nambu and G. Jona-Lasinio, Phys. Rev. 112 (1961), 345; Phys. Rev. 124, 246 (1961).

[58] M. K. Volkov, Ann. Phys.(N.Y.) 157, 282 (1984).

[59] T. Hatsuda and T. Kunihiro, Phys. Lett. B 145, 7 (1984).

[60] S. P. Klevansky, Rev. Mod. Phys. 64, 649, (1992).

[61] T. Hatsuda and T. Kunihiro, Phys. Rep. 247, 221 (1994).

[62] R. Alkofer, H. Reinhardt, and H.Weigel, Phys. Rep. 265, 239 (1996).

[63] M. Buballa, Phys. Rep.407, 205 (2005).

[64] P. Rehberg, S. P. Klevansky, and J. Hüfner, Phys. Rev. 
C 53, 410 (1995).

[65] G. S. F. Stephans and Y. Wu, J. Phys. G 23, 1895 (1997).

[66] T. A. Armstrong et al. (E864), Phys. Rev. C 59, 2699 (1999).

[67] B. B. Back et al. (E917), Phys. Rev. Lett. 87, 242301 (2001).
68] R. Rapp and E.V. Shuryak, Phys. Rev. Lett. 86, 2980 (2001).

[69] C. Greiner and S. Leupold, J. Phys. G 27, L95 (2001).

[70] L. McLerran, R. D. Pisarski, Nucl. Phys. A 837, 65 (2010]. 